\title{
OLIGOMER CHAIN LENGTH EFFECT ON THE NUCLEATION AND GROWTH MECHANISMS (NGM) OF POLYTHIOPHENE
}

\author{
M. ANGÉLICA DEL VALLE', MANUEL A. GACITÚA*', LUIS I. CANALES AND FERNANDO R. DÍAZI \\ ${ }^{\prime}$ Pontificia Universidad Católica de Chile. Facultad de Química, Departamento de Química Inorgánica, Laboratorio de Electroquímica de Polímeros. Av. \\ Vicuña Mackenna 4680, 7820436 Macul, Santiago, Chile. \\ (Received: January 19, 2009 - Accepted: May 28, 2009)
}

\begin{abstract}
In this research a comparative study of the starting unit chain length effect on the electropolymerization of thiophene or its oligomers was carried out considering mono (1Th), bi (2Th), ter (3Th), tetra (4Th) and sexi (6Th)-thiophene as starting units.

The deconvolved transient allows stating that the growth of polythiophene (PTh) employing each of the starting units have the same predominant contribution to the nucleation and growth mechanism (NGM). The others contributions disappear as the length of the starting unit chain increase. The results were validated by scanning electron microscopy (SEM) of PTh deposits obtained onto $\mathrm{SnO}_{2}$ coated glass, following a potential pulse program.

Besides of corroborating the electropolymerization model, this study suggested the possibility of designing and performing suitable experiments leading to the attainment of electro-deposited conductive polymers bearing a desired morphology, appropriated for prospective applications.
\end{abstract}

Keywords: Polythiophene, oligomer, nucleation and growth mechanism, electropolymerization

\section{INTRODUCTION}

In the last decades many papers has been released concerning to the preparation, characterization and applications of conducting polymers ${ }^{1-7}$. As synthetics metals, conductive polymers have gained interest among the scientist society due to their particular combined properties of metals (electrical behavior) and plastics (chemical resistance, reduced weight, simple formation processes) ${ }^{8}$. Moreover, polythiophene and its derivatives have been subject of studies concerning to physical, chemical, electronic and electrochemical properties, characterizing them as suitable electronic material for new technologies ${ }^{9-13}$

Its synthesis may be carried out through chemical or electrochemical strategies that considers the use of an oxidizing agent ${ }^{14,15}$ or an anodic perturbation, respectively ${ }^{16,17}$. With respect to the second approach, several aspects of the electropolimerization phenomena of polythiophene have been already elucidated. With regard to the NGM studies of the main experimental variable, about PTh electro-synthesis characterization, many challenges have been surmounted too ${ }^{18-20}$. Results showed that the growth mechanism of conducting polymers can be described from analogous studies effected on metallic deposits, but with the possibility of presenting more than one contribution to the global NGM. Factors such as the chemical nature and concentration of electrolyte and monomer, physical and chemical nature of the working electrode, solvent, and time of deposition determine which contribution prevails. However, there is no study concerning to the effect of the starting unit chain length as an experimental factor.

A proposed model, scheme 1 (a), for the electropolymerization phenomena has been reported elsewhere ${ }^{21}$.

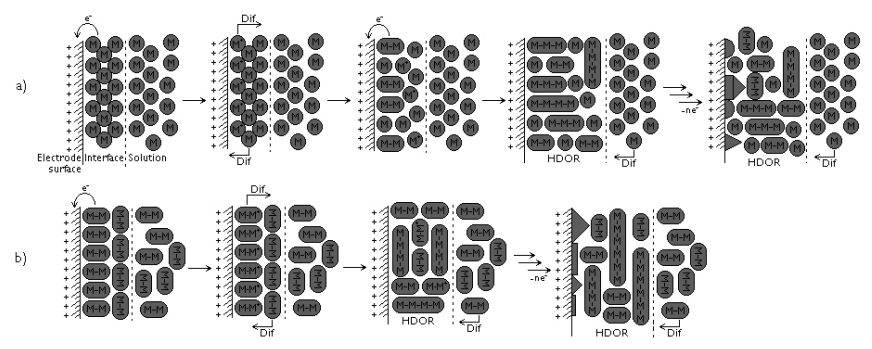

Scheme 1. Illustration of electropolymerization process considering a) $1 \mathrm{Th}$ and b) $2 \mathrm{Th}$ as the starting unit: Dif, diffusion; HDOR, high density oligomeric region.

There are also many papers about the electrochemical synthesis of PTh using thiophene known oligomers and its derivatives ${ }^{22-27}$. However there are currently few studies on the NGM of PTh using thiophene oligomers as starting unit. Recent cyclic voltammetric work has demonstrated that the polymerization potential decreases as longer thiophene chains are utilized. This has been ascribed to a higher level of conjugation.

If the same proposed model uses the dimer as starting unit, scheme 1 (b), the high density oligomeric region, HDOR, would be obtained earlier due to the formation of longer chains at the first stages of the oxidation. As for the NGM, it is expected that the contributions to the PTh polymerization from the monomer, should vary as follow:

1. Using longer oligomers as starting units produces longer chain fragments at the first oxidation stages, thereby the HDOR will be formed from components displaying less soluble and higher molecular mass. For example, $2 \mathrm{Th}$ produces $4 \mathrm{Th}$ and $6 \mathrm{Th} ; 3 \mathrm{Th}$ produces only $6 \mathrm{Th} . .$. etc.

2. When the supersaturation level is reached the precipitation of PTh onto the working electrode takes place by a less varied array of polymer fragments, compared to the deposition of the polymer when thiophene is used as starting unit.

3. Therefore, as the starting unit chain length increases the total number of contributions to the global NGM decreases, because the size variety of the depositing fragments is lower. Consequently, it should be expected that the contributions to the NGM would be more homogeneous to one type of mechanism.

4. Hence, it would be expected that the HDOR would resemble the bulk solution gradually until its disappearance when employing longer oligomers as starting units, because the chains without growing would be long enough to precipitate. Therefore, the diffusion controlled contributions to the global NGM should disappear while the charge transfer ones should prevail.

The purpose of this research was to test these hypotheses, thus the use of longer oligomers as starting units for the electropolymerization of PTh determines the appearance of less and more homogeneous contributions to the global NGM on a potentiostatic step program. Hence, synthesis of PTh was carried out following a systematic experimental path, scheme 2, explained in detail in the experimental.

\section{EXPERIMENTAL}

A polycrystalline non-annealed Pt disc (geometrical surface area 0.07 $\mathrm{cm}^{2}$ ) was used as working electrode. All potentials quoted in this paper are referred to a $\mathrm{Ag} / \mathrm{AgCl}$ electrode in tetramethylammonium chloride to match the potential of a SCE at room temperature. A platinum gauze of large geometrical area, separated from the cell main compartment by a fine glass sinter, was used as counter electrode.

Prior to each experiment the working electrode was polished with alumina slurry (particle size $0.3 \mu \mathrm{m}$ ) on soft leather and then rinsed with bi-distilled water, acetone, and finally dried. Electrochemical cell, syringe, volumetric flask and the rest of the employed glassware was always washed with bidistilled water, acetone, and oven-dried at $60{ }^{\circ} \mathrm{C}$ for at least $12 \mathrm{~h}$ prior to all experiments.

The supporting electrolyte employed was tetrabutylammonium hexafluorophosphate $\left(\mathrm{TBAPF}_{6}\right.$ ) supplied by Aldrich. When not in use the product was stored into an oven at $200{ }^{\circ} \mathrm{C}$ to prevent hydration. Thiophene, bithiophene, tertiophene, tetrathiophene, and sexithiophene were purchased 
from Aldrich. Dichloromethane (Aldrich, 99.99\% puriss) was stored in an inert atmosphere with dry argon and over molecular sieves $(4 \AA)$. To warrant a minimum water content, the solvent was handled with a syringe.

The solubility of $6 \mathrm{Th}$ in $\mathrm{CH}_{2} \mathrm{Cl}_{2}(2.5 \mathrm{mg} / 10 \mathrm{~mL})$ was chosen as the concentration of all starting units. This way the total number of heterocyclic units (thiophene) in solution is constant. Supporting electrolyte concentration was $0.1 \mathrm{M}$ in every case.

Prior to all experiments the solutions were purged with high purity argon and an argon atmosphere was maintained over the solution during the experiments.

Synthesis of polythiophene, using each oligomer, was carried out following the experimental procedure described in scheme 2.

\section{Cyclic Voltametry(CV); obtaining the CV profiles, the oxidation potential(OP) was found.}

\section{r}

Potentiostatic step at $\mathrm{OP}$; the obtained curves informed about the corresponding induction time, $\tau$.

\section{7}

Corrected current-time transient deconvolution; the NGM for each oligomer was proposed.

\section{r}

Potentiostatic pulses; Growth of a more homogeneous polymer film at different time pulses in $\mathrm{SnO}_{2}$ covered glass electrode

Scanning Electronic Microscopy (SEM); Photograph of the deposits and confirmation of the NGM proposed.

Scheme 2: Experimental Procedure.

All the electrochemical experiments were done at room temperature using an AUTOLAB PGSTAT20 potentiostat coupled to a PC running the AUTOLAB software which allowed the experimental control and data acquisition.

The SEM micrographs were obtained employing a LEO Electron Microscopy Inc. Model 1420VP

\section{RESULTS AND DISCUSSION}

3.1 Cyclic Voltammetry

Figure 1 shows the cyclic voltammetric profiles for each of the oligomers studied at the OP. This value was defined employing the following criteria:

A pronounced current increment is observed until OP is reached without change in the curve inflexion.

At this $\mathrm{OP}$ value a constant growth of the current from each $\mathrm{CV}$ profile is observed regarding the previous profile.

To prevent over-oxidation of the film, a reproducible stable response of each deposit was estimated on solution containing just the supporting electrolyte.

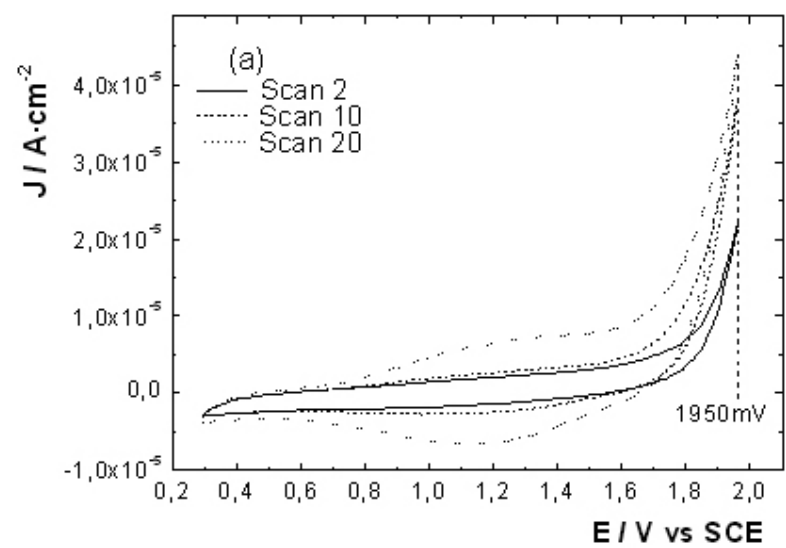

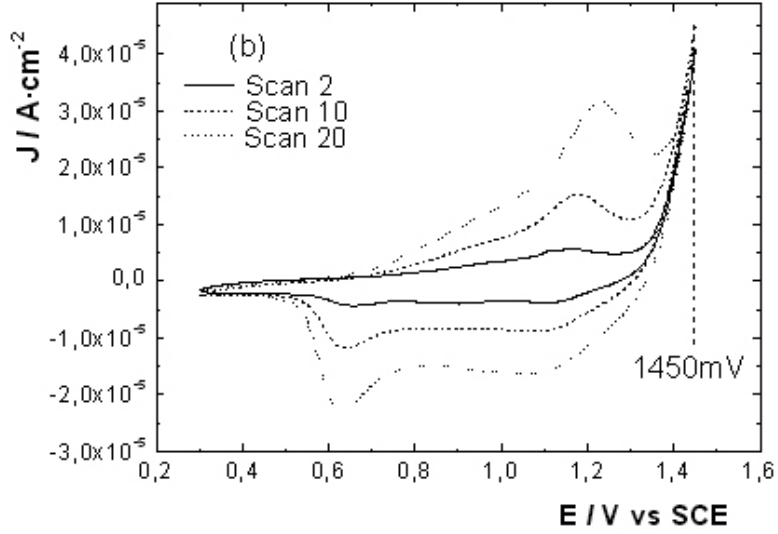
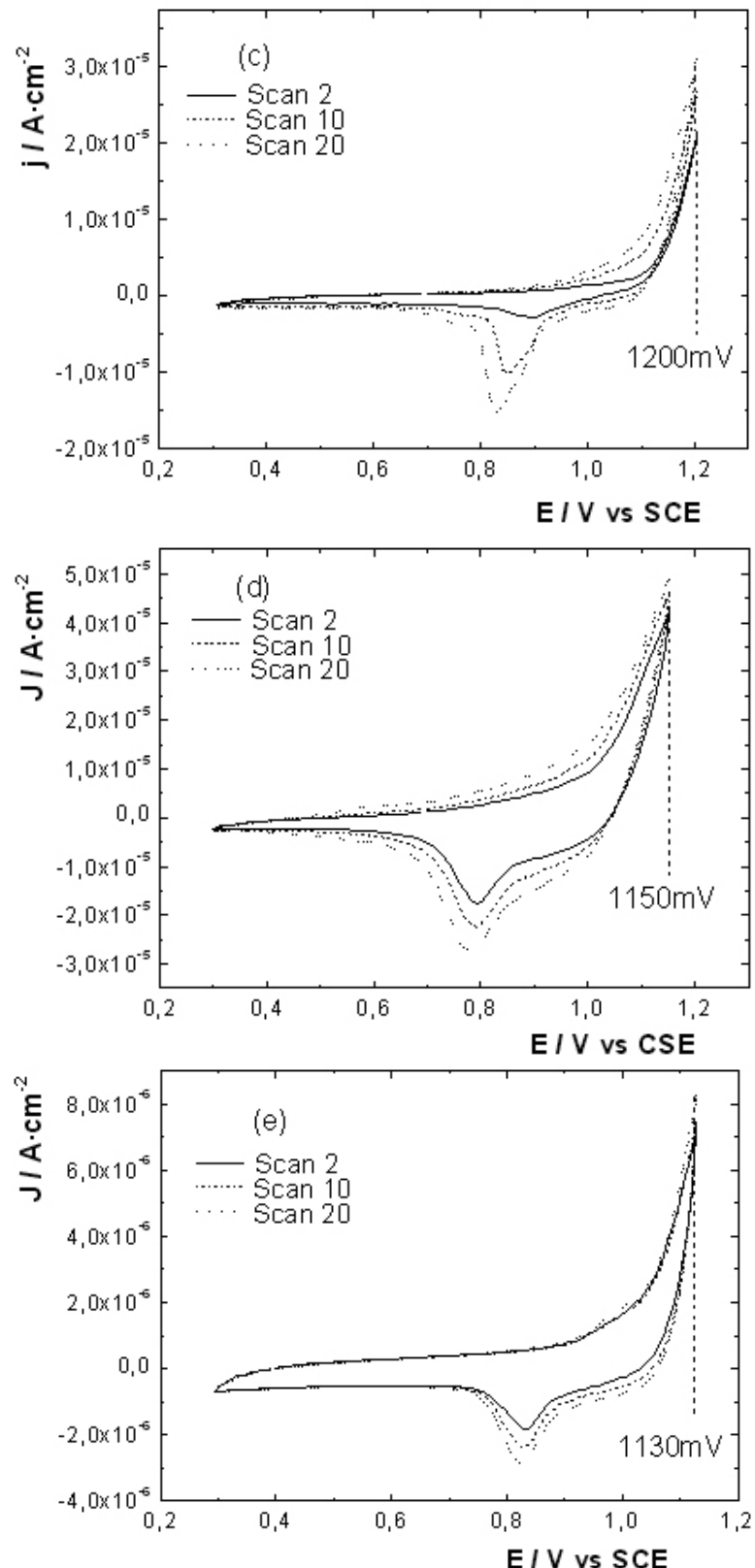

Figure 1: CV profiles. Interfase: Pt| (a) $3.0 \mathrm{mM} 1 \mathrm{Th}$, (b) $1.5 \mathrm{mM} 2 \mathrm{Th}$, (c) $1.0 \mathrm{mM} 3 \mathrm{Th}$ and (d) $0.75 \mathrm{mM} 4 \mathrm{Th}+0.1 \mathrm{M}$ TBAPF in $\mathrm{CH}_{2} \mathrm{Cl}_{2}$. Scan rate 100 $\mathrm{mV} \cdot \mathrm{s}^{-1}$ 
During the $\mathrm{CV}$ procedure formation of a deposit was observed, whose color changed from dark blue at the OP to light brown at the undoping potential. At the end of each $\mathrm{CV}$ process a blue-iridiscent deposit was obtained at the OP.

As the oligomer chain becomes longer, a decrease of the OP value was observed, which agrees closely with a higher level of conjugation. The higher the aromatic character of the starting unit the easier the oxidation proceeds.

This is clear evidence that the electropolymerization of PTh has been successfully accomplished by using the respective CV program at the selected OP.

3.2 Potentiostatic steps

Utilizing the same experimental conditions as above, a potentiostatic step method at the polymerization potential, PP, was conducted.

Current-time transients (Fig. 2) were obtained. The PP used in each case was selected from the potential range, at the $\mathrm{CV}$ profiles, where formation of the most conducting film occurs.
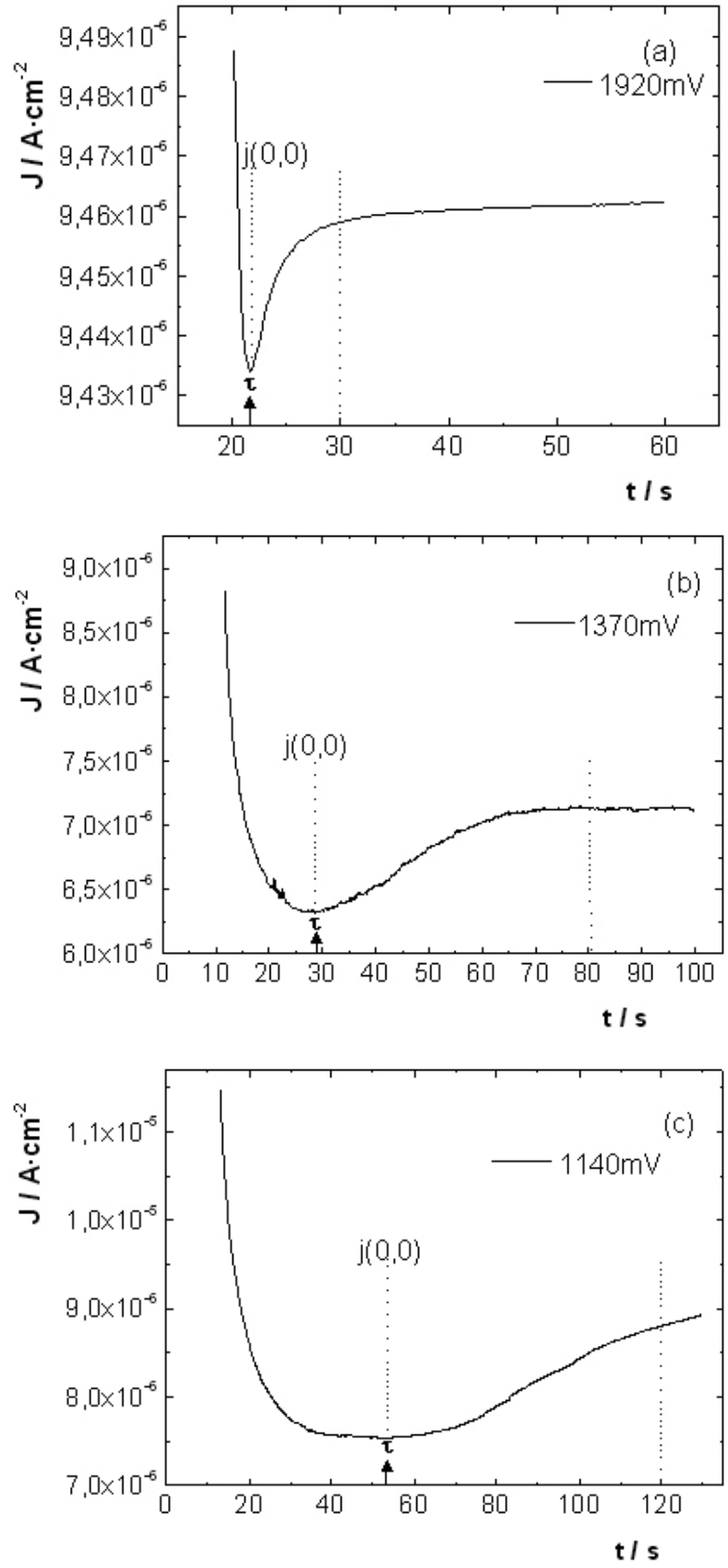



Figure 2: Current-time transients. Interfase: same as Figure 1. (a) 1Th, (b) 2Th, (c) $3 \mathrm{Th}$ and (d) $4 \mathrm{Th}$

The induction time, $\tau$, is known as the time necessary to exceed the saturation level of oligomer at the electrode solution interfase. This phenomenon describes the HDOR formation, whose existence has been demonstrated in earlier studies. Once the super-saturation level is exceeded the deposition of PTh takes place. A proper current-time transient for $6 \mathrm{Th}$ could not be obtained due to the poor adherence of the synthesized oligomers on the working electrode surface. Consequently, no polymer growth is obtained. A reasonable explanation will be given below.

$\tau$ values for $1 \mathrm{Th}, 2 \mathrm{Th}, 3 \mathrm{Th}$, and $4 \mathrm{Th}$ were $21.8,28.8,54.7$, and 28.4 respectively. A clear trend of these values was not observed. It must be pointed out that the molar concentration was not constant in every case. The molar concentrations of the oligomer series were $3.0,1.5,1.0,0.75$, and $0.5 \mathrm{mM}$, respectively. As stated in the experimental, the study considered constant the mass amount but not the concentration. This way the total number of heterocycles is the same for each experience, allowing to studying the effect of the number of heterocyclic units per chain on the NGM, independently of its concentration. To this end, the solubility of sexithiophene in $\mathrm{CH}_{2} \mathrm{Cl}_{2}$ $(2.5 \mathrm{mg} / 10 \mathrm{~mL})$ was chosen as the constant mass content in every case. As the induction time varies directly with the initial molar concentration of the reactive species and the supersaturation level is more easily achieved by longer oligomeric chains, no definite trend in the $\tau$ values is expected.

In order to study the NGM, the current-time transients have been corrected using only the fragment between the limits depicted on each curve. The considered part represents the formation of the first nuclei, at $\mathrm{j}(0,0)$, and their growth, excluding the diffusional contribution of the monomer oxidation.

$3.3 N G M$

Figure 3 shows the deconvolved corrected current-time transients. 

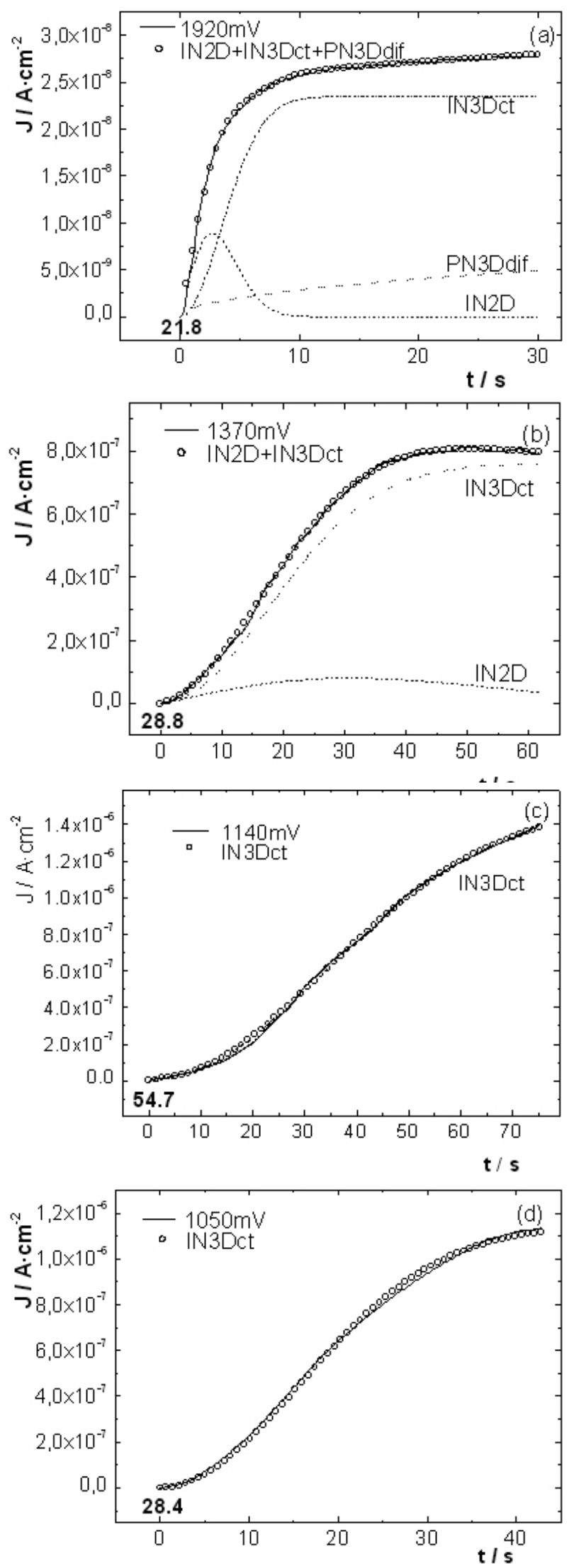

Figure 3: Deconvolved corrected current-time transients. (a) 1Th, (b) $2 \mathrm{Th}$, (c) $3 \mathrm{Th}$ and (d) $4 \mathrm{Th}$.
As previously reported ${ }^{18,20}$, the deconvolved transients show that the NGM of PTh is described by more than one contribution. Each contribution to the net charge, as previously demonstrated, varies with time, and the length of the starting unit chain determines the number of contributions. For 1Th bidimensional instantaneous nucleation (IN2D), three-dimensional instantaneous nucleation controlled by charge transfer (IN3D), and three-dimensional progressive nucleation controlled by diffusion $\left(\mathrm{PN}^{\mathrm{C}} \mathrm{D}_{\mathrm{dif}}\right)$ contributions are observed. When the starting unit is $2 \mathrm{Th}$ the $\mathrm{PN} 3 \mathrm{D}_{\text {dif }}$ contribution disappears. The IN2D contribution for $1 \mathrm{Th}$ reaches its maximum before the one on $2 \mathrm{Th}$, which is explained by the mayor molar concentration of starting unit on the first case. Finally, for PTh with 3Th and 4Th as starting unit, the sole contribution to the NGM was IN3D . The above discussion explains why for oligomer fragments formed using 6Th as starting unit lack of bidimensional growth at the first stages of nucleation and necessarily leads to less adherent films onto the surface of the working electrode, making heavier oligomer fragments, from the HDOR, to fall into the solution. Consequently, no suitable current-time transient for the electropolymerization of sexithiophene was obtained, although blue solid fragments of the polymer fell off from the Pt disk, going into the solution.

As the length of the starting unit chain increase, a decrease in the number of contributions and a predominance of instantaneous nucleation was observed. This behavior will be discussed below.

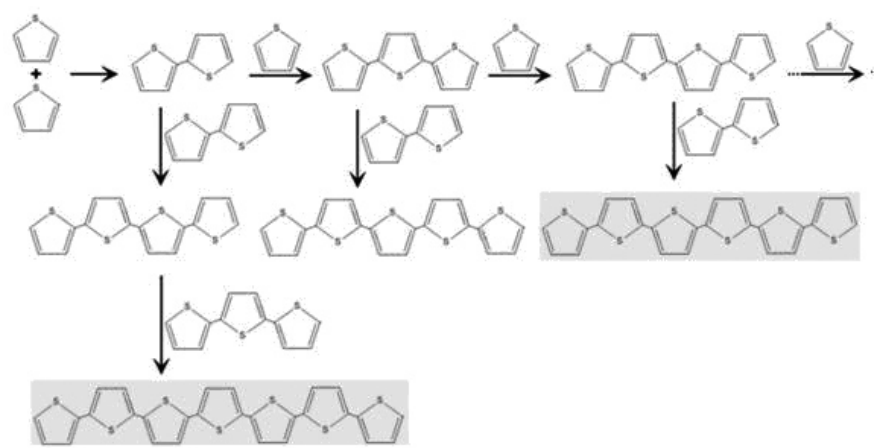

Scheme 3: 1Th chain reactions.

Studies regarding deposition of PTh onto the working electrode, employing 3Th as starting unit, have demonstrated that the main structure formed during the oxidation is the six-membered oligomer, $6 \mathrm{Th}^{28}$. This suggests that polymer fragments larger than sexithiophene will also precipitate onto the working electrode during the anodic perturbation. Scheme 3 shows the different likely reactions of the monomer, 1Th, during the anodic perturbation. After formation of the HDOR, the two different highlighted structures will precipitate onto the working electrode. If the same scheme is thought for $2 \mathrm{Th}, 3 \mathrm{Th}, 4 \mathrm{Th}$, and $6 \mathrm{Th}$ as staring units, the number of probable reactions decreases because the effective formation of longer chains would take place by the first reaction (scheme 3). Consequently, precipitation of less varied structures occur. Also, the formation and growth of nuclei would be accomplished by the precipitation of these polymer fragments, leading the NGM of PTh that presents less and more homogeneous contributions along the oligomeric series. To confirm the obtained results and the posed hypotheses, a morphological study of the deposits was performed.

\subsection{SEM}

Using the same experimental conditions as above, but using fluorine doped $\mathrm{SnO}$ coated glass (CG) as working electrode, potentiostatic pulses programs at the polymerization potential, were applied. Program setups are depicted in Fig. 5. 



Figure 5: Potentiostatic pulses programs setup. (a) $2 \mathrm{~s}$, (b) $10 \mathrm{~s}$ and (c) $30 \mathrm{~s}$ time pulse.

It has been verified that this change on the electrodic substrate doesn't imply differences on the global NGM for the electropolymerization ${ }^{29}$. In fact, only small differences on the induction time value, $\tau$, has been observed which are negligible when correlating the growth mechanism with deposit morphology.

Depending on the NMG obtained from the deconvolved corrected current-time transients, program (a), (b) and/or (c) was chosen as setup for a potentiostatic-pulse program applied to each starting unit. The morphology of the obtained deposit should reveal if the proposed NGM was right.

SEM was employed to study the morphology of the PTh deposited onto CG. Figure 6 shows the micrographs of the deposits obtained for $1 \mathrm{Th}$.

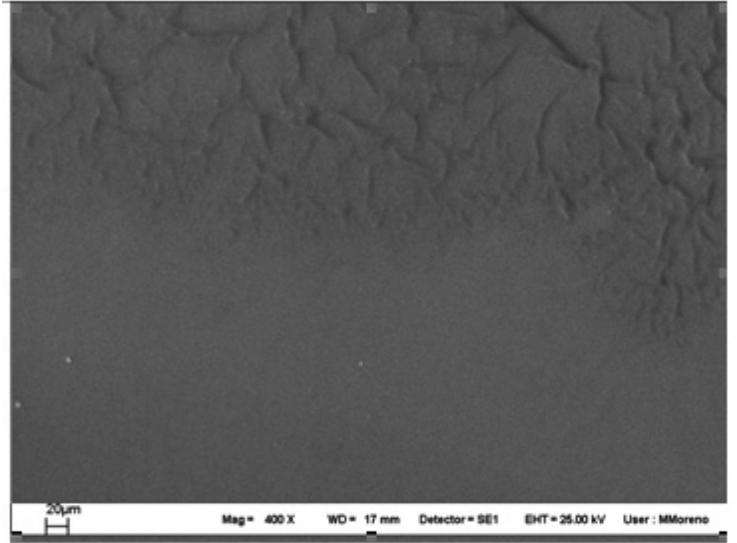

(a)

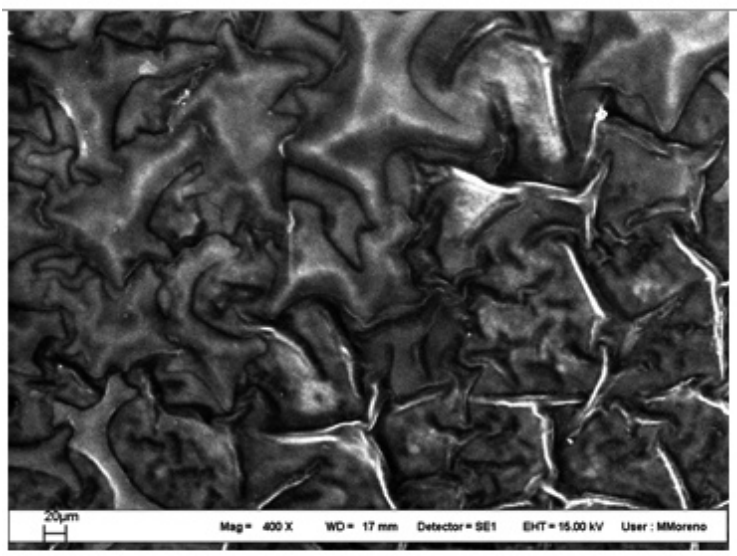

(b)

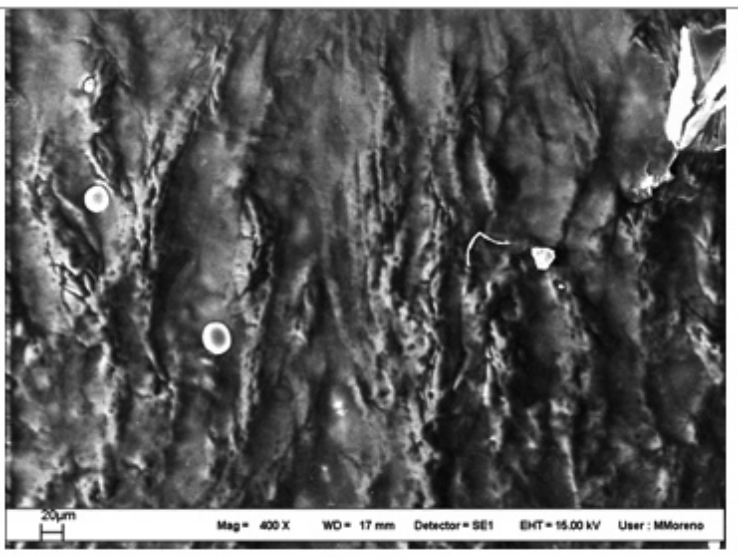

(c)

Figure 6: SEM Micrographs of PTh deposits from 1Th onto CG . For (a), (b) and (c) the program employed was 2, 10, and 30 s pulse, respectively.

The NMG of PTh, using 1Th as the starting unit, Fig. 4a, clearly shows that at two seconds of the anodic perturbation the main contribution to the growth mechanism is IN2D. Therefore, the growth of PTh would follow a cylindrical shape pattern and if many grown nuclei are formed by several potentiostatic pulses, a uniform deposit on the electrode will be expected. Therefore, Fig. 6 (a) represents this situation.

At ten seconds IN $3 \mathrm{D}_{\mathrm{ct}}$ mainly contributes to the net charge of the transient with a small contribution of $\mathrm{PN} 3 \mathrm{D}_{\text {dif }}$. This contribution follows a conical shape. 
Figure 6 (b) may not exactly represent a conical morphology, but the deposit shows a degree of roughness higher than in (a). This reveals the existence of contributions other than IN2D.

Micrograph (c) show a deposit with similar grade of roughness but with higher grade of coverage. However, no typical morphology might be found. At 30 seconds the main contributions to the NGM of PTh are IN3Dct and PN3Ddif, describing irregular forms and shapes of deposits. This way the proposed NGM of PTh using 1Th as the starting couldn't be confirmed but the result are consistent with a higher grade of roughness and coverage as the pulse time increase.



Figure 7: SEM Micrograph of PTh deposits from 2Th onto CG. The program employed was $2 \mathrm{~s}$.

At $2 \mathrm{~s}$ the main contribution to the NGM of PTh, using $2 \mathrm{Th}$ as the starting unit (figure $4 \mathrm{~b}$ ), is IN2D. Figure 7 clearly represent the growth of PTh by a bidimensional mechanism, showing an uniform morphology. Unfortunately none appropriate deposit was attained employing the 30s pulse. During experimentation a poor adherence of PTh, with $2 \mathrm{Th}$ as the starting unit on CG was achieved. The superficial part of the deposit was released when the working electrode was removed from the solution and, consequently, the original morphology was modified.

Experiments performed with 3Th, 4Th, and 6Th as starting unit considered a different approach. Instead of removing the glass electrode from the cell, the remained solution was flushed with Ar, leaving the deposit practically intact.



(a)

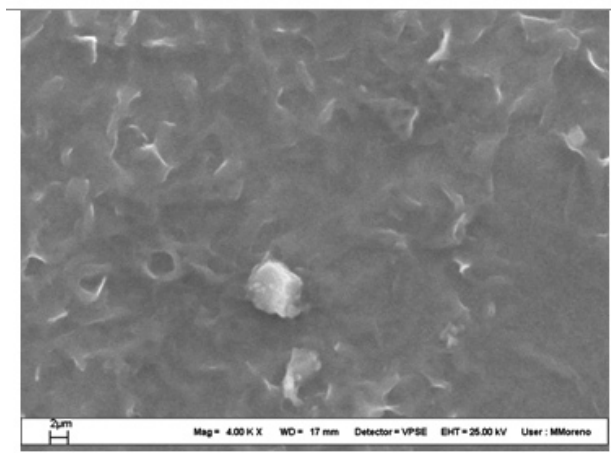

(b)

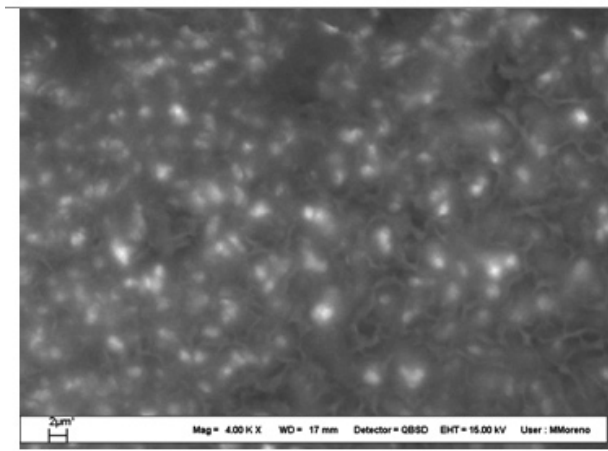

(c)

Figure 8: SEM Micrographs of PTh deposits from 3Th onto CG. For (a) and (b) the program employed was $2 \mathrm{~s}$; for (c) $30 \mathrm{~s}$ pulse, respectively.

Figure 8 shows micrographs of the polymer deposit using 3 Th as starting unit. The corresponding NGM for this case is shown in Fig. 4 (c). Micrograph (a) shows a homogeneous deposit with some grain like formation on the electrode surface. A closer look, at (b) reveals the presence of spike-like formations. This caused by the film dryness after a few days of storage.

Micrograph (c) show the presence of several growth nuclei with a pointy end. Therefore, IN3D mechanism produces several conical growth nuclei onto the surface and the $30 \mathrm{~s}$ time pulse program achieves to demonstrate the NGM proposed.

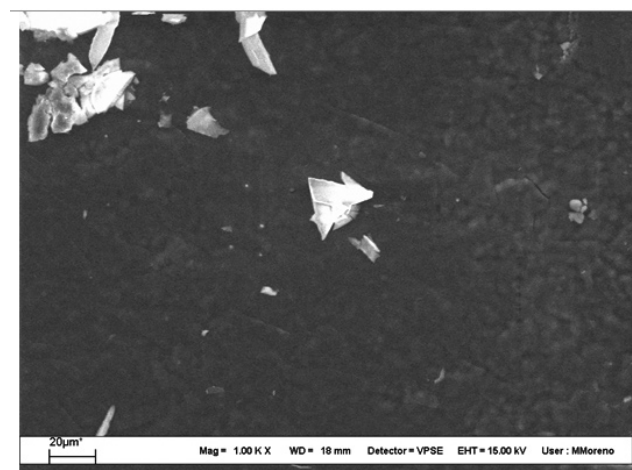

(a)

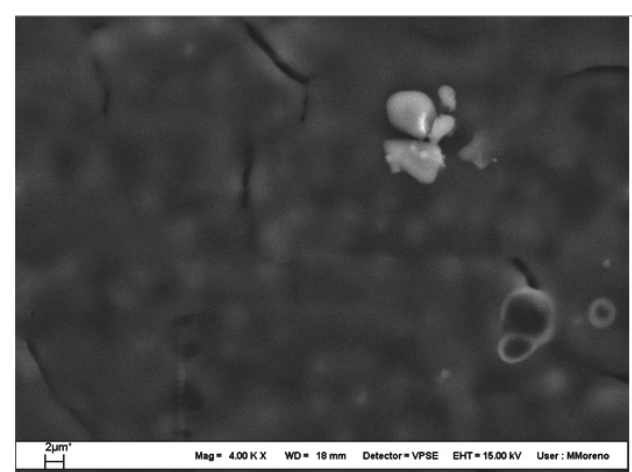

(b)

Figure 9: SEM Micrographs of PTh deposits from 4Th onto CG. The program employed was $30 \mathrm{~s}$ time pulse.

Figure 9 shows micrographs of the polymer deposit using 4Th as starting unit. The NGM for this case is depicted in Fig. 4(d). Both micrographs show a homogeneous granulated deposit on the electrode surface but with an apparent higher thickness of the film than the deposits made from 3Th. This could indicate that the same NGM is confirmed. However, no clear conclusion can 
be stated about the mechanism confirmation due to the unclear thick deposit morphology.



(a)

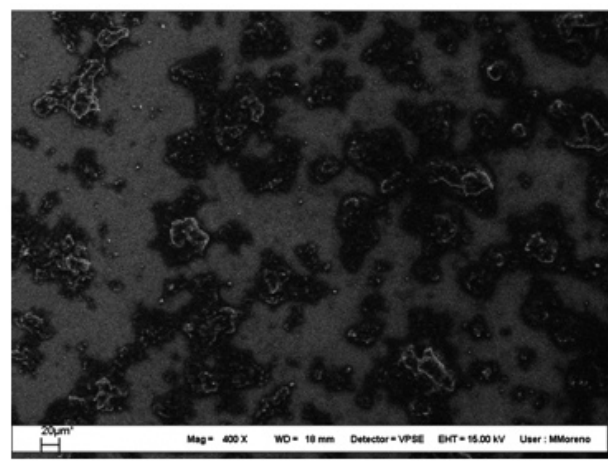

(b)

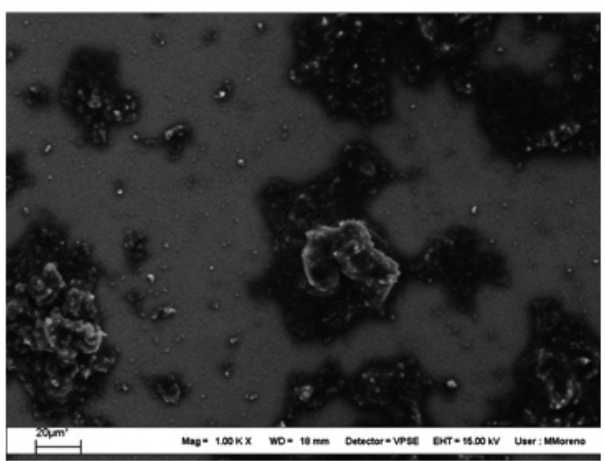

(c)

Figure 10: SEM Micrographs of PTh deposits from 6Th onto CG. A 2s time pulse program was employed for (a) and (b); for (c) 30 s.

Using sexithiophene as starting unit, no PTh deposition was observed on Pt disk electrodes, but an appreciable amount of solid was attained when a $\mathrm{SnO}_{2}$ coated glass was employed as substrate. The reason might be that the roughness of this kind of surface provides better adherence to the oligomers, which favors PTh deposition. However, 6Th, being a highly complex and heavy molecule, needs not form the 12-membered molecule in order to precipitate. Thus, the deposition occurs mainly onto the first formed growth nuclei and no deposit bearing a regular structure is expected, as evidenced in the respective micrographs.

\section{CONCLUSIONS}

Cyclic voltammetry studies have demonstrated that as the starting unit chain length increases, the OP decreases responding to the higher aromatic character of the longer units. The current-time transient curves presented no clear trend of the estimated $\tau$ values, due to the abovementioned experimental conditions on the experimental section.

The results validate the PTh NGM model previously attained proposed elsewhere ${ }^{21}$, where more than one contribution to the net charge of a corrected deconvolved current-time transient was determined. However, the number of contributions to the net charge decreases with the length of the oligomer employed as the starting unit (scheme 1). Also the IN3D $\mathrm{D}_{\mathrm{ct}}$ contribution prevails along the oligomers series. This behavior responds to the deposition of polymer fragments from the HDOR region explained in scheme 1 and 3.

SEM micrographs of the deposits reveal that employing PP programs at different time pulse lengths will produce certain morphology determined by the NGM's contributions only on certain cases with a high grade difficulty. The results, in some cases, have demonstrated the mechanism proposed for the growth of PTh from the different starting unit, allowing in advance to state the working conditions in order to obtain the desired specific deposit morphology.

\section{ACKNOLEDGEMENTS}

The authors thank Comisión Nacional de Investigación, Ciencia y Tecnología (CONICYT) trough grant Fondecyt \# 1060598.

\section{REFERENCES}

1. L. Chang, C. Yen, J. Appl. Polym. Sci. 55, 371, (1994)

2. L. Wang, X. Li, Y. Yang, Reac. Func. Polym. 47, 125, (2001)

3. J. D. Stenger Smith, Prog. Polym. Sci. 23, 57, (1998)

4. F. Brovelli, B. Rivas, J. C. Bernède, J. Chil. Chem. Soc. 50, 597, (2005).

5. C. San Martín, P. Dreyse, C. García, K. Calfumán, D. Villagra, M. Isaacs, J. Chil. Chem. Soc. 52, 1305, (2007).

6. A. S. Ribeiro, W. A. Gazotti, V. C. Nogueira, D. A. Machado, P. F. dos Santos Filho, M. A. De Paoli, J. Chil. Chem. Soc. 49, 197, (2004).

7. J. C. Bernède, J. Chil. Chem. Soc. 53, 1549, (2008).

8. E. Hakansson, K. Kaynak, T. Lin, S. Nahavandi, T. Jones, E. Hu, Synth. Met. 144, 21, (2004)

9. R. Schrebler, M. A. del Valle, H. Gómez, C. Veas, R. Córdova, J. Electroanal. Chem. 380, 219, (1995)

10. M. E. Nicho, H. Hailin, C. López-Mata, J. Escalante, Sol. Energy. Mater. Sol. Cells. 82, 105, (2004)

11. P. Dreyse, G. Ramírez, A. Riquelme, M. Isaacs, J. Chil. Chem. Soc. 51, 923, (2006)

12. F. Brovelli, B. Rivas, J. C. Bernède, J. Chil. Chem. Soc. 52 (2007).

13. S. Torres, G. Neculqueo, F. Martínez, J. Chil. Chem. Soc. 52, 1235 , (2007).

14. P. Haaland, J. Targove, Appl. Phys. Lett. 61, 34, (1992)

15. P. Enzel, T. Bein, J. Chem. Soc: Chem. Comm. 1326, (1989)

16. M. A. del Valle, F. R. Díaz, M. E. Bodini, T. Pizarro, R. Córdova, H. Gómez, R. Schrebler, J. Appl. Electrochem. 28, 943, (1998)

17. P. Blanchard, L. Huchet, E. Levillain, J. Roncali, Electrochem. Comm. 2 , $1,(2000)$

18. R. Schrebler, P. Grez, P. Cury, C. Veas, M. Merino, H. Gómez, R. Córdova, M. A. del Valle, J. Electroanal. Chem. 430, 77, (1997)

19. M. A. del Valle, P. Cury, R. Schrebler, Electrochim. Acta 48, 397, (2002)

20. M. A. del Valle, F. R. Díaz, F. Bustos, L. Guerra, J. Chil. Chem. Soc. 48, 23, (2003).

21. M. A. del Valle, F. R. Díaz, M. E. Bodini, G. Alfonso, G. M. Soto, E. Borrego, Polym. Int. 54, 526, (2005)

22. E. A. Bazzaoui, S. Aeiyach, P. C. Lacaze, Synth. Met. 83, 159, (1996)

23. Y. Wei, C. Chan, J. Tian, G. Jang, K.F. Hsueh, Chem. Mater. 3, 888, (1991)

24. B. Krische, M. Zagorska, Synth. Met. 35, 257, (1989)

25. S. J. Higgins, T. J. Pounds, P. A. Christensen, J. Mater. Chem. 11, 2253, (2001)

26. A. S. Sarac, U. Evans, M. Serantoni, V.J. Cunnane, Carbon. 41, 2725, (2003)

27. E. Brillas, R. Oliver, F. Estrany, E. Rodríguez, S. Tejero, Electrochim. Acta. 47, 1623, (2002)

28. Z. Xu, D. Fichou, G. Horowitz, F. Garnier, J. Electroanal. Chem. 267, $339,(1989)$

29. M. A. del Valle, M. B. Camarada, F. R. Díaz, G. East, e-Polymers 072 , $1,(2008)$ 